\title{
Evaluation of beam halo from beam-gas scattering at the KEK Accelerator Test Facility
}

\author{
R. Yang, ${ }^{1, *}$ T. Naito, ${ }^{2,3}$ S. Bai, ${ }^{4}$ A. Aryshev, ${ }^{2,3}$ K. Kubo, ${ }^{2,3}$ T. Okugi, ${ }^{2,3}$ N. Terunuma, ${ }^{2,3}$ \\ D. Zhou, ${ }^{2,3}$ A. Faus-Golfe, ${ }^{1}$ V. Kubytskyi, ${ }^{1}$ S. Liu, ${ }^{5}$ S. Wallon, ${ }^{1}$ and P. Bambade ${ }^{1}$ \\ ${ }^{1}$ LAL, Univ. Paris-Sud, CNRS/IN2P3, Université Paris-Saclay, Orsay 91898, France \\ ${ }^{2}$ High Energy Accelerator Research Organization, Tsukuba, Ibaraki 305-0801, Japan \\ ${ }^{3}$ School of High Energy Accelerator Science, SOKENDAI, Tsukuba, Ibaraki 305-0801, Japan \\ ${ }^{4}$ Institute of High Energy Physics, Beijing 100049, China \\ ${ }^{5}$ Deutsches Elektronen-Synchrotron, Hamburg D-22607, Germany
}

(Received 14 March 2018; published 23 May 2018)

\begin{abstract}
In circular colliders, as well as in damping rings and synchrotron radiation light sources, beam halo is one of the critical issues limiting the performance as well as potentially causing component damage and activation. It is imperative to clearly understand the mechanisms that lead to halo formation and to test the available theoretical models. Elastic beam-gas scattering can drive particles to large oscillation amplitudes and be a potential source of beam halo. In this paper, numerical estimation and Monte Carlo simulations of this process at the ATF of KEK are presented. Experimental measurements of beam halo in the ATF2 beam line using a diamond sensor detector are also described, which clearly demonstrate the influence of the beam-gas scattering process on the transverse halo distribution.
\end{abstract}

DOI: 10.1103/PhysRevAccelBeams.21.051001

\section{INTRODUCTION}

In high-energy lepton colliders, the balance between the requirements of high luminosity and low detector backgrounds is always a challenge. To control the background induced by halo particles with large betatron amplitude or energy deviation, a robust collimation system is essential. The design of collimators requires some knowledge of the halo distribution and population, to estimate the collimation efficiency [1]. To describe the halo distribution and mechanisms for its formation, a number of numerical and experimental investigations have been performed, for both circular and linear machines [2-6]. These studies indicate that halo distributions are influenced by many factors, e.g., space charge, scattering (elastic and inelastic beam-gas scattering, intrabeam scattering and $e^{-}$cloud), optical mismatch, chromaticity, and optical aberrations. Moreover, the dominant halo source might be different for each machine, depending on its design and status.

For the future linear colliders, it is essential to determine plausible halo distributions at the entrance of the main linac and their physical origin. The Accelerator Test Facility (ATF) of KEK, which has successfully achieved small

\footnotetext{
*ryang@1al.in2p3.fr
}

Published by the American Physical Society under the terms of the Creative Commons Attribution 4.0 International license. Further distribution of this work must maintain attribution to the author(s) and the published article's title, journal citation, and DOI. emittances satisfying the requirements of the International Linear Collider (ILC), and which includes an extraction line (ATF2) capable of focusing the beam down to a few tens of nanometers at the virtual interaction point (IP), is an ideal machine to study halo formation mechanisms and develop the specialized instrumentation needed for the measurements. At the ATF2 beam line, the reduction of the modulation in the beam size measurement using the Shintake monitor [7] at the IP due to halo loss upstream also motivates a good understanding of the halo formation and ways to suppress it. Considerable efforts have been devoted to reveal the primary mechanism controlling halo formation at ATF [8-11]. The theory to characterize beam profile diffusion due to elastic beam-gas scattering (BGS) has been developed, but has not yet been fully validated experimentally, mainly due to the lack of appropriate instrumentation with high enough dynamic range (DNR, $\geq 10^{5}$ ). To achieve a suffcient DNR, a set of diamond sensor detectors (DS) has been constructed and installed at the end of the ATF2 beam line [12].

In this paper, numerical evaluations of beam halo from BGS are described, followed by a detailed simulation of halo formation in the presence of radiation damping, quantum excitation, residual dispersion, $x y$ coupling and BGS in the damping ring. Halo measurements using the diamond sensor detector are described, which confirm that the vertical halo is dominated by BGS. The results are then discussed and some conclusions and further work are outlined. 


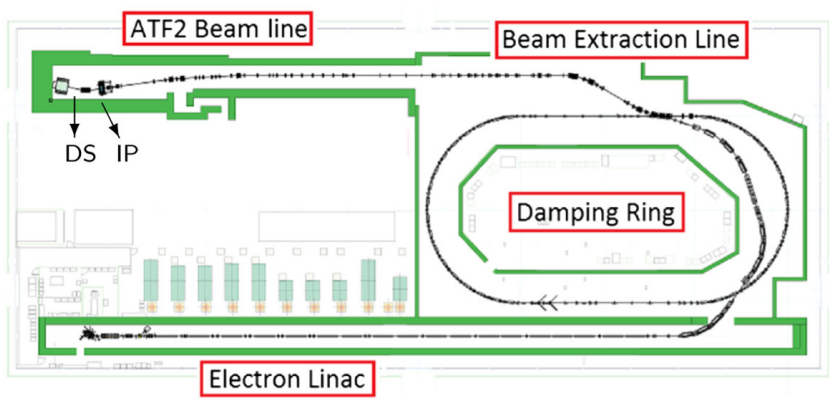

FIG. 1. Schematic diagram of ATF linac, damping ring and ATF2 beam line, from [14].

\section{A. Accelerator Test Facility 2}

ATF consists of a $1.3 \mathrm{GeV} S$-band linac, a damping ring and an extraction line, as shown in Fig. 1. The smallest vertical rms emittance measured at low intensity was $4 \mathrm{pm}$ [13], which corresponds to the normalized emittance of $1.1 \times 10^{-8} \mathrm{~m}$. The main beam parameters in the ATF damping ring are summarized in Table I.

As an extension of ATF, ATF2 aims to address the feasibility of focusing the beam to a few tens of nanometer size and providing beam orbit stabilization at the nanometer level at the IP. ATF2 is also an energy-scaled version of the compact focusing optics designed for the ILC, using a similar local chromaticity correction scheme $[17,18]$.

\section{THEORETICAL EVALUATION}

\section{A. Analytic approximations}

We follow the approach developed by K. Hirata [8] for the description of particle redistribution in the presence of stochastic processes. The transverse motion in a ring or transport beam line can be perturbed by stochastic processes such as synchrotron radiation, BGS or IBS. It can be described by the diffusion equation

$$
\frac{d \vec{x}}{d s}=-[H(\vec{x}, s), \vec{x}]+\xi(\vec{x}, s)
$$

TABLE I. ATF main parameters $[15,16]$.

\begin{tabular}{lcc}
\hline \hline Beam energy [GeV] & $E_{0}$ & 1.3 \\
Circumference [m] & $C$ & 138.6 \\
Intensity [e/pulse] & $N$ & $1-10 \times 10^{9}$ \\
Vertical emittance [pm] & $\epsilon_{y}$ & $>4$ \\
Horizontal emittance [nm] & $\epsilon_{x}$ & 1.2 \\
Energy spread [\%] & $\sigma_{\delta}$ & $0.056(0.08)^{\mathrm{a}}$ \\
Bunch length [mm] & $\sigma_{z}$ & $5.3(7)^{\mathrm{a}}$ \\
Damping time [ms] & $\tau_{x} / \tau_{y} / \tau_{z}$ & $17 / 27 / 20$ \\
Injection emittance [nm] & $\epsilon_{x 0} / \epsilon_{y 0}$ & 14 \\
Storage time [ms] & $t$ & 200 \\
Momentum acceptance [\%] & $\Delta p / p$ & 1.2 \\
\hline \hline
\end{tabular}

${ }^{a}$ With intra-beam scattering (IBS) for the beam intensity of $1 \times 10^{10} \mathrm{e} /$ pulse. where $\vec{x}$ is the $6 \mathrm{D}$ phase space coordinate, $H(\vec{x}, s)$ the Hamiltonian representing the symplectic part of the motion and $\xi(\vec{x}, s)$ contains the diffusion effects. The solution to the equation of motion can be expressed in terms of a linear map plus the integrated perturbation of the stochastic process

$$
\vec{x}(s)=M\left(s, s_{0}\right) \vec{x}_{0}+\int_{s_{0}}^{s} M\left(s, s^{\prime}\right) \tilde{\xi}(s) d s^{\prime}
$$

with

$$
M\left(s, s_{0}\right)=M_{0} \exp \left[\int_{s_{0}}^{s}\left[s \tilde{H}\left(s^{\prime \prime}\right)-D\left(s^{\prime \prime}\right)\right] d s^{\prime \prime}\right]
$$

where $M_{0}$ is the symplectic matrix representing the linear transformation, $\tilde{H}$ a symmetric $6 \times 6$ matrix and $D$ the damping matrix which contains the radiation damping [19]. Here we describe only the transverse motion (in the horizontal plane for example) and we consider only the betatron motion, radiation damping, quantum excitation and diffusion from BGS, ignoring betatron coupling. In normalized coordinates $u=x / \sqrt{\beta}$ and $u^{\prime}=d u / d \phi$, Eq. (2) can be written

$$
\vec{u}(s)=R\left(s, s_{0}\right) \vec{u}\left(s_{0}\right) \exp \left(-\frac{\alpha}{c_{0}} \int_{s_{0}}^{s} d s\right)+\delta \vec{u}
$$

where $\vec{u}=\left(u, u^{\prime}\right)^{T}, R\left(s, s_{0}\right)$ is a pure rotation, $\alpha$ is the damping rate and $\delta \vec{u}$ the perturbation, expressed as

$$
\begin{gathered}
R\left(s, s_{0}\right)=\left(\begin{array}{cc}
\cos (\Delta \phi) & \sin (\Delta \phi) \\
-\sin (\Delta \phi) & \cos (\Delta \phi)
\end{array}\right) \\
\delta \vec{u}=R\left(s, s_{0}\right)\left(\begin{array}{c}
0 \\
\sqrt{\beta} \theta_{x}
\end{array}\right) \exp \left(-\frac{\alpha}{c_{0}} \int_{s_{0}}^{s} d s\right)
\end{gathered}
$$

where $\Delta \phi=\int_{s_{0}}^{s} \frac{d s}{\beta(s)}$ is the phase advance, $\beta$ the betatron function, $\theta_{x}$ the transverse kick angle at $s_{0}$ and $c_{0}$ light velocity in vacuum. We can further specify the perturbation term in Eq. (4) in terms of the transformation in presence of radiation damping, diffusion due to the quantum excitation, $\delta \vec{u}_{q e}$, and the external perturbation due to BGS, $\delta \vec{u}_{e x}$

$$
\vec{u}(s)=R\left(s, s_{0}\right) \vec{u}\left(s_{0}\right) \exp \left(-\frac{\alpha}{c_{0}} \int_{s_{0}}^{s} d s\right)+\delta \vec{u}_{q e}+\delta \vec{u}_{e x}
$$

The stationary distribution is determined by the integral of all stochastic processes. Since particle distributions under the influence of radiation damping and quantum excitation are well understood, it is convenient to express the distribution function $\psi(u)$ as 


$$
\psi(u)=\frac{1}{2 \pi} \int e^{i \omega u} \tilde{\psi}_{t}(\omega) \tilde{\psi}_{f}(\omega) d \omega
$$

where $\tilde{\psi}_{t}(\omega)$ is the characteristic function in the presence of radiation damping and quantum excitation

$$
\tilde{\psi}_{t}(\omega)=\exp \left(-\omega^{2} \sigma_{t}^{2} / 2\right)
$$

and $\sigma_{t}$ is the beam size in absence of external perturbation. The characteristic function $\tilde{\psi}_{f}(\omega)$ has been derived in Refs. [8] and [20], thanks to Campbell's theorem [21]. Here, we use the formalism in Ref. [8] where the stochastic perturbation is treated over many betatron oscillation periods. Approximating $\beta$ by its average value over the ring, $\bar{\beta}$, the characteristic function $\tilde{\psi}_{f}(\omega)$ can be written as

$$
\tilde{\psi}_{f}(\omega)=\exp \left[\frac{N}{\alpha} \hat{f}(\omega \sqrt{\bar{\beta}})\right]
$$

where

$$
\hat{f}(\tilde{\omega})=\frac{2}{\pi} \int_{0}^{1} d \zeta \frac{\Re[\tilde{f}(\tilde{\omega} \zeta)]-1}{\zeta} \cos ^{-1} \zeta
$$

and

$$
\tilde{f}(\tilde{\omega})=\int d \theta_{x} f\left(\theta_{x}\right) \cos \left(\tilde{\omega} \theta_{x}\right)
$$

The factor $N$ is the scattering rate of a test particle, $\Re[\tilde{f}(\tilde{\omega} \zeta)]$ the real part of $\tilde{f}(\tilde{\omega} \zeta)$ and $f\left(\theta_{x}\right)$ is the probability distribution for a deflection angle $\theta_{x}$. The final distribution function can be expressed as

$\psi(u)=\frac{1}{2 \pi} \int_{-\infty}^{\infty} e^{i \omega u} \exp \left[-\frac{\omega^{2} \sigma_{t}^{2}}{2}+\frac{N}{\alpha} \hat{f}(\omega \sqrt{\bar{\beta}})\right] d \omega$

This characteristic function is an even function, so only the cosine part remains after performing the integration

$\psi(u)=\frac{1}{\pi} \int_{0}^{\infty} \cos (\omega u) \exp \left[-\frac{\omega^{2} \sigma_{t}^{2}}{2}+\frac{N}{\alpha} \hat{f}(\omega \sqrt{\bar{\beta}})\right] d \omega$

The transverse distribution in $x$ can be described by

$\psi\left(x_{i}\right)=\frac{1}{\pi} \int_{0}^{\infty} \cos \left(\omega x_{i}\right) \exp \left[-\frac{\omega^{2} \sigma_{x_{i}}^{2}}{2}+\frac{N}{\alpha} \hat{f}\left(\omega \sqrt{\bar{\beta} \beta_{i}}\right)\right] d \omega$

where $x_{i}$ is the horizontal coordinate at position $i, \sigma_{x_{i}}$ the equilibrium horizontal beam size in presence of radiation damping and quantum excitation, and $\beta_{i}$ is the beta function at the observation point.
To obtain the numerical form of the distribution function $\psi(u)$ or $\psi(x)$, we have to first evaluate $\tilde{f}(\tilde{\omega})$ in the presence of BGS. Treating BGS as the classical Rutherford scattering process and considering the screening effect, the cross section in the CGS system of unit is given by

$$
\frac{d \sigma}{d \Omega}=\left(\frac{2 Z r_{e}}{\gamma}\right)^{2} \frac{1}{\left(\theta^{2}+\theta_{m}^{2}\right)^{2}}
$$

where $\Omega$ is the solid angle, $Z$ the atomic number, $r_{e}$ the classical electron radius, $\gamma$ the Lorentz factor, $\theta$ the transverse deflection angle and $\theta_{m}$ the minimum angle due to electron shielding

$$
\theta_{m}=\frac{\alpha_{0} Z^{1 / 3}}{\gamma}
$$

where $\alpha_{0}$ is the fine structure constant. The transverse deflection angle $\theta$ can be further specified as

$$
\theta^{2}=\theta_{x}^{2}+\theta_{y}^{2}
$$

Note $\theta_{x} \in\left[-\theta_{x, \max }, \theta_{x, \max }\right]$ and the same for $\theta_{y}$. The differential $d \sigma / d \theta_{x}$ can be obtained by integration of Eq. (16) over the vertical deflection angle $\theta_{y}$. If we assume $\theta_{y, \max } \gg \sqrt{\theta_{x}^{2}+\theta_{m}^{2}}, d \sigma / d \theta_{x}$ can be approximated by

$$
\frac{d \sigma}{d \theta_{x}} \approx \frac{\pi}{2}\left(\frac{2 Z r_{e}}{\gamma}\right)^{2} \frac{1}{\left(\theta_{x}^{2}+\theta_{m}^{2}\right)^{3 / 2}} .
$$

Then the total cross section $\sigma_{\text {tot }}$, probability function $f\left(\theta_{x}\right)$ and scattering rate $N$ become

$$
\begin{gathered}
\sigma_{\mathrm{tot}}=\int_{-\theta_{x, \max }}^{\theta_{x, \max }} \frac{d \sigma}{d \theta_{x}} d \theta_{x}=\frac{4 \pi Z^{2} r_{e}^{2}}{\gamma^{2} \theta_{m}^{2}} \\
f\left(\theta_{x}\right)=\frac{1}{\sigma_{\mathrm{tot}}} \frac{d \sigma}{d \theta_{x}}=\frac{\theta_{m}^{2}}{2\left(\theta_{x}^{2}+\theta_{m}^{2}\right)^{3 / 2}} \\
N=\rho_{v} \sigma_{\mathrm{tot}} c_{0}
\end{gathered}
$$

where $\rho_{v}$ is the volume density of residual gas atoms. Following the derivation in Ref. [8], functions $\tilde{f}(\tilde{\omega})$ and $\hat{f}(\tilde{\omega})$ are finally expressed as

$$
\begin{aligned}
& \tilde{f}(\tilde{\omega})=\tilde{\omega} K_{1}(\tilde{\omega}) \\
& \hat{f}(\tilde{\omega})=\frac{2}{\pi} \int_{0}^{1} d \zeta \frac{\tilde{\omega} \zeta K_{1}(\tilde{\omega} \zeta)-1}{\zeta} \cos ^{-1} \zeta
\end{aligned}
$$

where $K_{1}$ is the modified Bessel function of first order. Estimates of the beam profile using Eq. (15) for the ATF damping ring are shown in Fig. 4. 

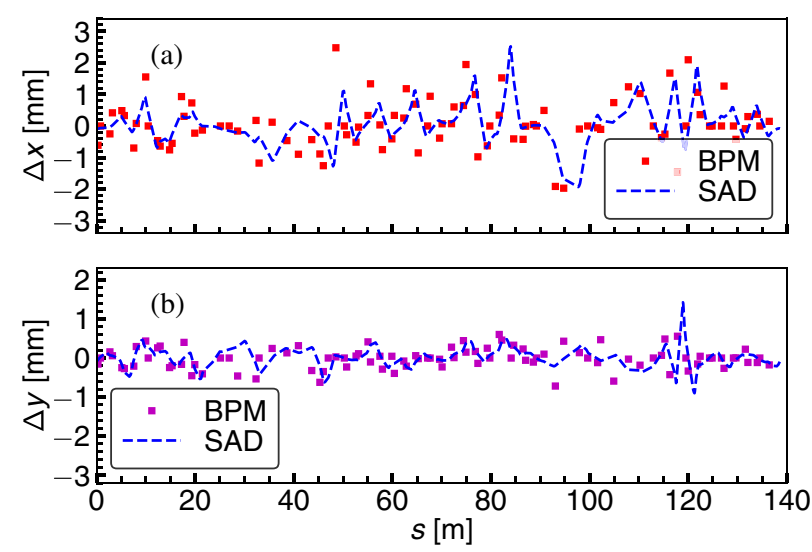

FIG. 2. Horizontal (a) and vertical (b) COD measured by BPMs in January 2017 and approached by local orbit bumps.

\section{B. Tracking simulations}

Generation and tracking of core particles and scattered particles were performed through a script developed in SAD [22], a program used for optical matching and closed-orbit distortion (COD) correction during beam operation. The equilibrium vertical emittance $\epsilon_{y}$ is mainly determined by the residual vertical dispersion $\eta_{y}$ and cross-plane betatron coupling, both of which strongly depend on the magnet alignment errors and the resulting COD [15,23]. The vertical emittance can be modeled by introducing random vertical displacements to quadrupoles and sextupoles (20 $\mu \mathrm{m}$, RMS), and rotations of quadrupoles (2 mrad, RMS). The equilibrium emittance $\epsilon_{y}$, obtained for various seeds ranges from $5 \mathrm{pm}$ to $30 \mathrm{pm}$. Alternatively, the actual COD measured by BPMs can be modeled by local orbit bumps using steering magnets, as shown in Fig. 2. Equilibrium emittances are $12 \mathrm{pm}$ and $1.2 \mathrm{~nm}$, vertically and horizontally, respectively, for a realistic COD. The latter can better represent the realistic orbit and beam parameters, and is therefore used in our BGS simulations. The emittances and beam sizes considered here and in the following are evaluated by Gaussian fits to the beam core distributions.

Tracking of both scattered and non-scattered particles is performed element-by-element utilizing the beam parameters at injection shown in Table I. The simulation of scattered particles is performed as follows [24]. First, in each turn, the number of scattering events and their perturbations are generated randomly according to the residual gas pressure and the cross section. Second, perturbations in the $6 \mathrm{D}$ phase space of particles are implemented at random longitudinal positions to simulate particle scattering. The location of particle scattering is approximated to be at the closest element, which determines the local Twiss parameters and orbit. Third, the scattered particles in the present turn are transported to the observation point (at the location of the extraction kicker), to be combined with the scattered particles accumulated from the previous turns. The above process is then repeated until beam extraction. In addition, the possibility of multiBGS has been considered.

In order to estimate beam profile in the ATF 2 beam line, stored particles are extracted and transported to diagnostic points. Twiss parameters of the ATF2 lattice are well matched with the damping ring lattice at the extraction kicker. Orbit distortion of the extracted beam in the kicker-septum region can be represented by a coordinate transformation. The " $10 \times 1$ " optics [14] of the ATF2, with beta-functions of $\beta_{x}=40 \mathrm{~mm}$ and $\beta_{y}=0.1 \mathrm{~mm}$ at the IP, is used.

Estimates of the vacuum lifetime $\tau_{v}$, which depends directly on the gas pressure, supply benchmarks for the simulations. We assume $Z=\sqrt{50}$ and two atoms per molecule, which approximates air or CO [8], to represent the residual gas. For an average gas pressure of $1 \times 10^{-6} \mathrm{~Pa}$, the calculated value of $\tau_{v}$ is $83 \mathrm{~min}$ [25]. Meanwhile, the simulated value is $87 \mathrm{~min}$ using the equilibrium beam parameter and realistic physical apertures.

Vacuum beam lifetime was also measured at ATF, assuming the beam lifetime is dominated by Touschek scattering and elastic BGS. The time dependence of the beam intensity can be described by

$n(t)=1-\alpha \int_{0}^{t} d t^{\prime} P\left(t^{\prime}\right) n\left(t^{\prime}\right)-\frac{1}{\tau_{\text {Tou }}(\kappa)} \int_{0}^{t} n^{2}\left(t^{\prime}\right) d t^{\prime}$

where $n(t)=N(t) / N_{0}$ is the normalized beam intensity, $\alpha=1 /\left(\tau_{v} P\right)$ a coefficient related to the vacuum lifetime $\tau_{v}$ and gas pressure $P$, and $\tau_{\text {Tou }}$ is the Touschek lifetime. The decay of the beam current and the variation of the average gas pressure are shown in Fig. 3 for different vertical emittances. The coefficient $\alpha$ is around $1000 \mathrm{~Pa}^{-1} \mathrm{~s}^{-1}$, and $\tau_{v} \approx 16 \mathrm{~min}$, as determined by fitting the current decay with
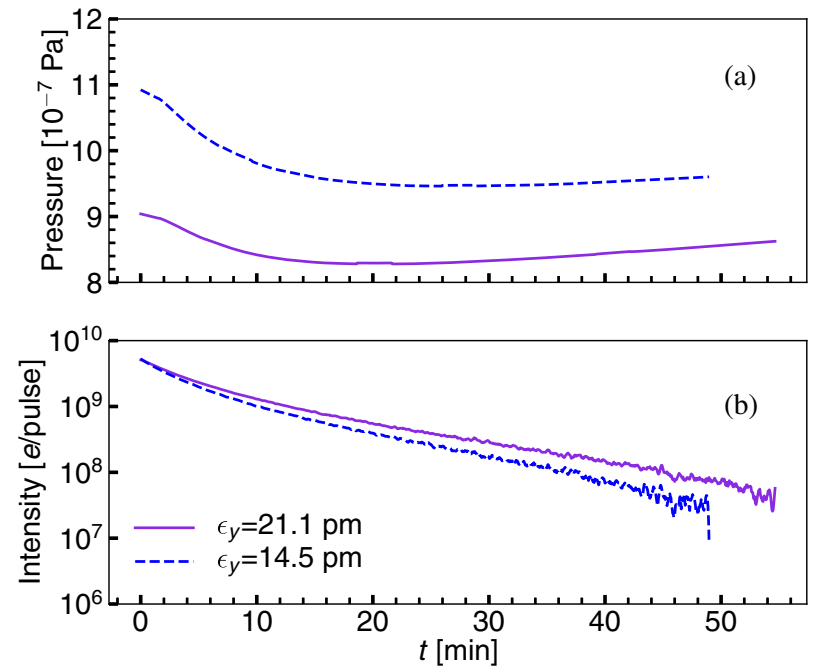

FIG. 3. Evolution of the averaged gas pressure (a) and current decay of the stored beam (b) in the ATF damping ring. 

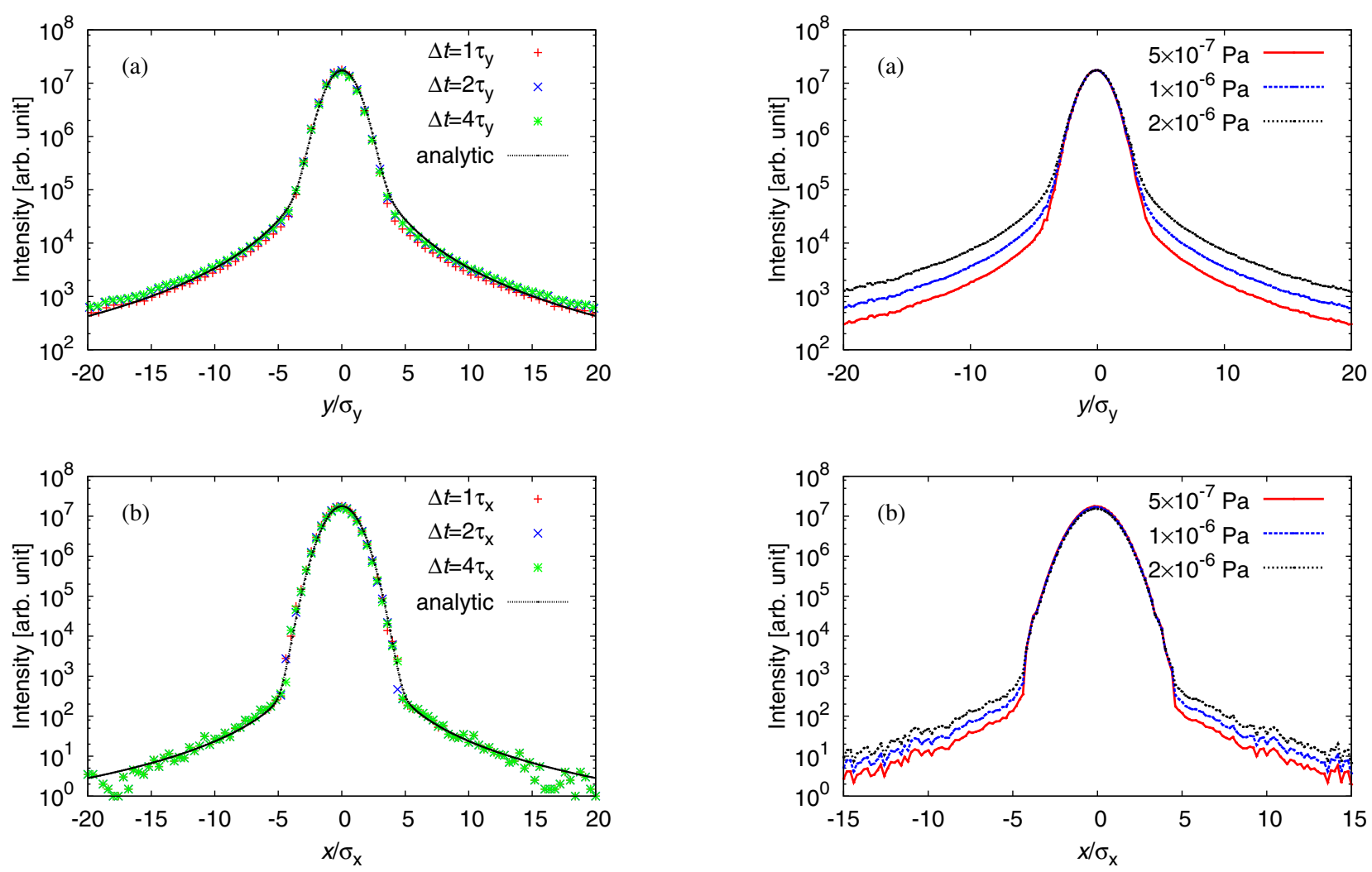

FIG. 4. Comparison of vertical (a) and horizontal (b) beam distortion between analytic approximation and simulation. A tracking time of more than 2 damping times is essential to reach the equilibrium.

Eq. (24). Such a reduction in the experimentally measured vacuum lifetime has been reported in Ref. [26] and Ref. [27], which suggest the probable beam loss channels: (1) existence of a larger horizontal beam halo induced by other mechanisms; (2) reduction of the dynamic aperture due to sextupole components at the entrance/exit of the combined function bending magnets.

The cross section of elastic beam-gas scattering is inversely proportional to $\theta^{2}$ and therefore the large-angle events are infrequent. Thus, we set an upper bound on the scattering angle at $100 \theta_{m}$, which is much larger than the RMS divergence of core particles. The minimum angle $\theta_{m}$ for the ATF beam is $5.5 \mu \mathrm{rad}$. To acquire sufficient statistics, the number of accumulated particle scattering events can be as many as $2 \times 10^{7}$. These simulations indicate that at least twice the damping time is essential to reach the equilibrium distribution in the ATF damping ring. For the typical vacuum level of $5 \times 10^{-7} \mathrm{~Pa}$, satisfactory agreement between the analytical calculation using Eq. (15) and the simulation is observed (see Fig. 4), where the distribution is normalized to the core beam size. After such a normalization, the horizontal tail/halo appears lower than the vertical halo by around two orders of magnitude, due to the flat aspect ratio of the ATF beam, the horizontal beam size being typically ten times larger than the vertical.

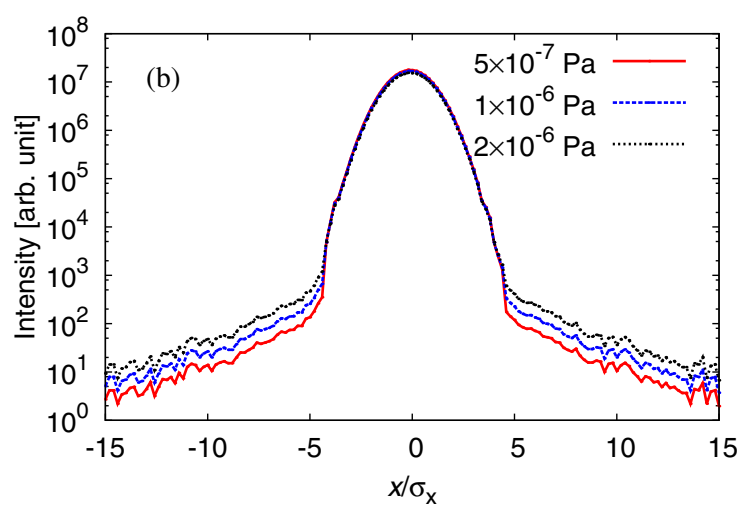

FIG. 5. Dependence of vertical (a) and horizontal (b) beam profiles on vacuum pressure.

The probability of BGS depends on the density of residual molecules, and therefore, the beam halo can increase for higher vacuum pressure in the ring. Presently, the average gas pressure obtained in the normal operation is $2 \times 10^{-7} \mathrm{~Pa}$, which can be adjusted by turning off some of the sputtering ions pumps (SIPs). Simulations have been performed for three different pressure levels which were achieved in operation. Significant increases of the beam tail/halo can be observed for higher vacuum pressure, as shown in Fig. 5.

\section{EXPERIMENTAL HALO MEASUREMENTS}

\section{A. Experimental setup and procedures}

Two beam halo detectors based on chemical vapor deposition (CVD) single crystal diamond sensors have been built and installed after the IP. Each diamond sensor is $500 \mu \mathrm{m}$ thick, with the metalization arranged in four strips, two broad ones with the dimensions of $1.5 \mathrm{~mm} \times 4 \mathrm{~mm}$ and two narrow ones of $0.1 \mathrm{~mm} \times 4 \mathrm{~mm}$. The strips and related circuitry are mounted on a ceramic printed circuit board (PCB) and placed in vacuum. All the strips are biased at $-400 \mathrm{~V}$ and connected to $50 \Omega$ resistors by coaxial cables for signal readout by an oscilloscope, as shown in Fig. 6. To suppress high frequency noise on the supplied bias voltage and to provide a sufficient reserve of charge for the largest signals, a low-pass filter together with charging 


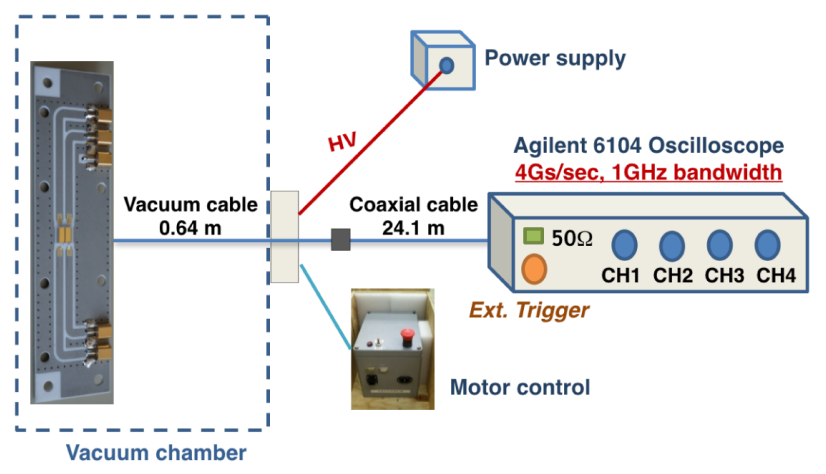

FIG. 6. Layout of diamond sensor on ceramic PCB (left) and the data acquisition system (right).

capacitors are mounted on the backside of the ceramic PCB [12]. Since the DS are located behind a large bending magnet, the horizontal dispersion is close to $1 \mathrm{~m}$ for the " $10 \times 1$ " optics.

The linear dynamic range of the diamond was demonstrated to be $10^{4}$, with a lower limit of $10^{3}$ electrons, which is mainly determined by pickup noise induced by the passage of the beam in the vicinity, and a linear response up to $2 \times 10^{7}$ electrons, which is limited by charge collection saturation effects in the diamond. Since a few thousand electrons is acceptable as background noise for the preliminary halo measurement, emphasis was put on the suppression of the saturation effect for the large signals. In the beam core region, the readout becomes nonlinear and the waveform can be strongly distorted both due to space charge inside the diamond crystal bulk and to the instantaneous voltage drop in the $50 \Omega$ resistor, as shown in Fig. 7(a). The response of the output signal with respect to the charge collected by the DS strip is shown in Fig. 7(b). The number of electrons striking the diamond can be evaluated according to the beam intensity and transverse beam size, although this can involve some uncertainties due to the instabilities at high intensity.

Rather than reconstructing the waveform based on the charge collection dynamics [28], a "self-calibration" method was proposed to enable suitable correction of the core profile. In this case, the beam core distribution could be measured by a wire scanner (WS) located $2.89 \mathrm{~m}$ upstream and propagated to the DS to predict the number of electrons striking each strip according to its position with respect to the beam center. Subsequently, the charge $Q_{\exp }$ which would be collected in the absence of saturation was computed based on the known electron hole pairs generation and charge collection efficiency measured at low incident charge [12]. The rescaling factor $\kappa$ was then defined as the ratio of $Q_{\text {exp }}$ to the charge signal readout, and applied to rescale the DS data within beam core. After such rescaling based on "self-calibration," the linear dynamic range could be extended beyond $10^{5}$ for the populations of collected electrons ranging from $1 \times 10^{3}$ to
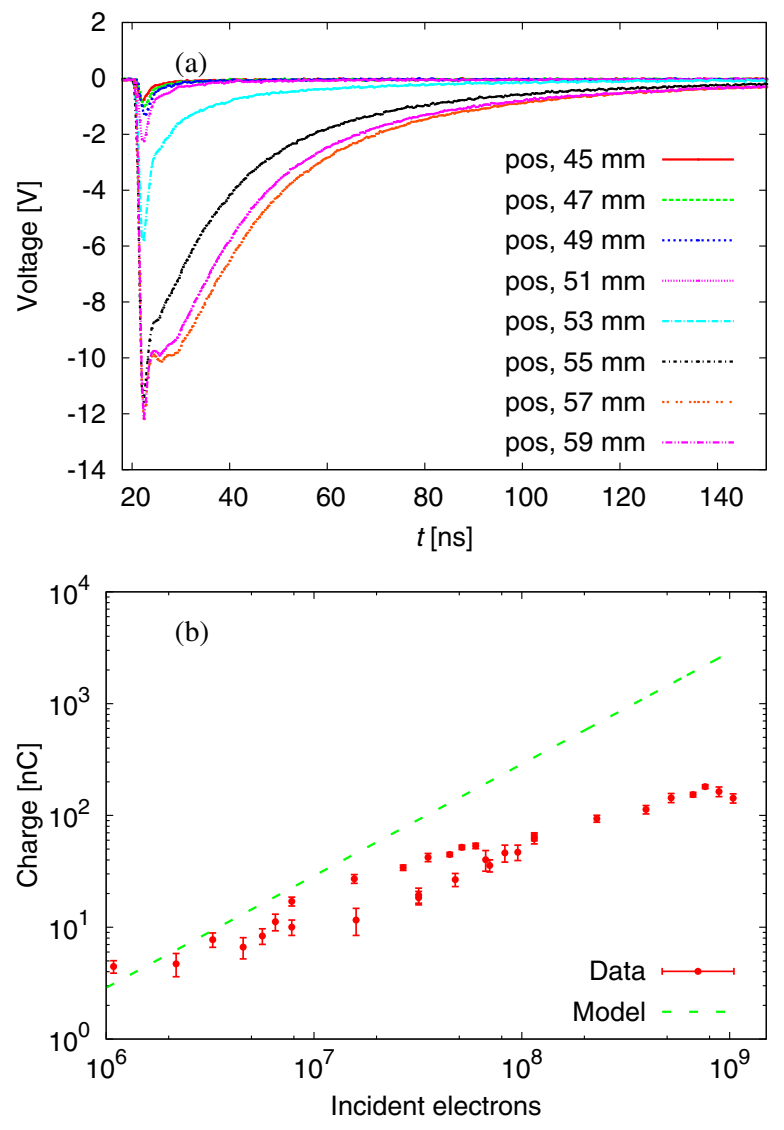

FIG. 7. Typical waveforms measured with the DS within the core region where beam center at around $57 \mathrm{~mm}$ (a) and charge signal as a function of the quantity of collected electrons (b).

more than $5 \times 10^{8}$. The corrected beam profile is shown in Fig. 8.

\section{B. Transverse beam distribution}

The transverse beam halo was measured using the DS for various vacuum pressures in the ATF damping ring. Beam intensity was stabilized at $3 \times 10^{-9} e /$ pulse, and the residual gas pressure was increased by switching off SIPs in the arc sections and north straight section of the ATF damping ring.

Measured vertical beam halo distributions, after implementing the rescaling corrections, are consistent with predictions from tracking simulations, as shown in Fig. 9(a). Moreover, the enhancement of the vertical halo for degraded vacuum pressures is clearly observed. Good agreement between simulations and experiments indicates that the dominant mechanism for vertical halo formation is elastic BGS in the ring.

The measured horizontal beam distributions were also corrected using the described self-calibration method. The reconstructed beam profiles are higher than the predictions from BGS and asymmetrical distributions are observed, with more halo particles on the right side (high energy 

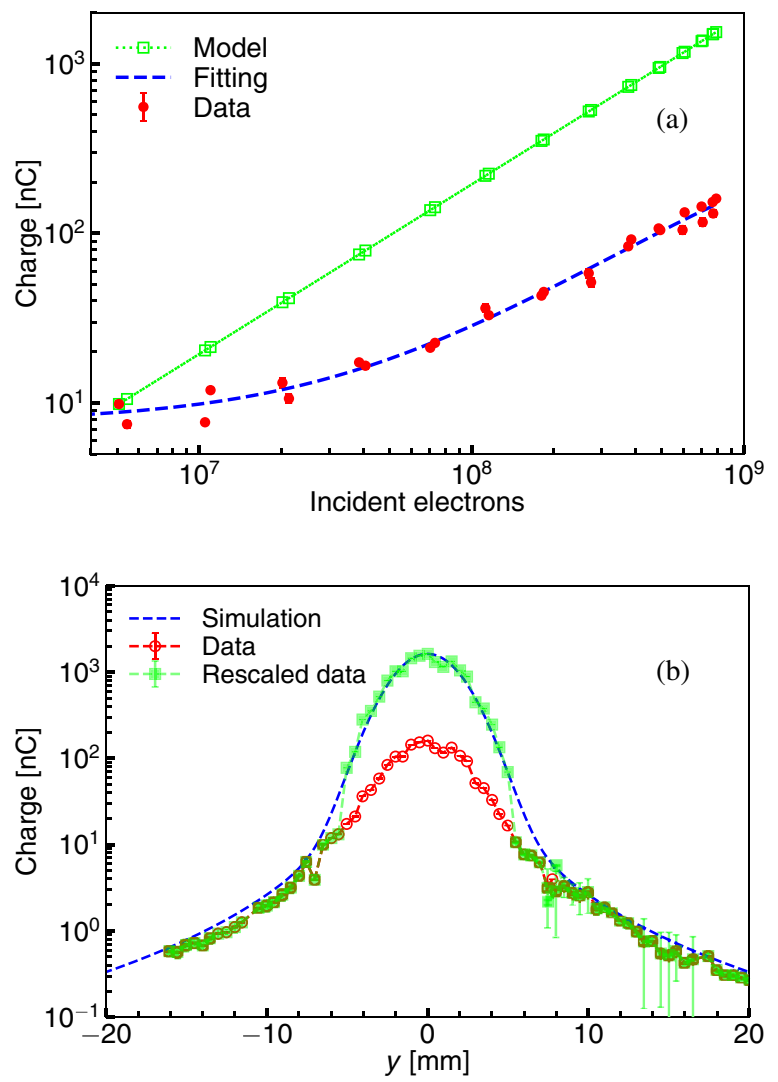

FIG. 8. Ideal and actual charge collections on the DS strip as a function of incident electron population for the evaluation of rescaling factor (a) and comparison of vertical beam profile before and after correction (b).

side), as shown in Fig. 9(b). In addition, the evolution of the beam halo with the vacuum level was found to be negligible, which might be due to insufficient sensitivity, since the background noise level is around $0.01 \mathrm{nC}$. The DS being located in a high dispersion region after a large horizontal bending magnet $\left(\eta_{x} \approx 1 \mathrm{~m}\right)$, potential

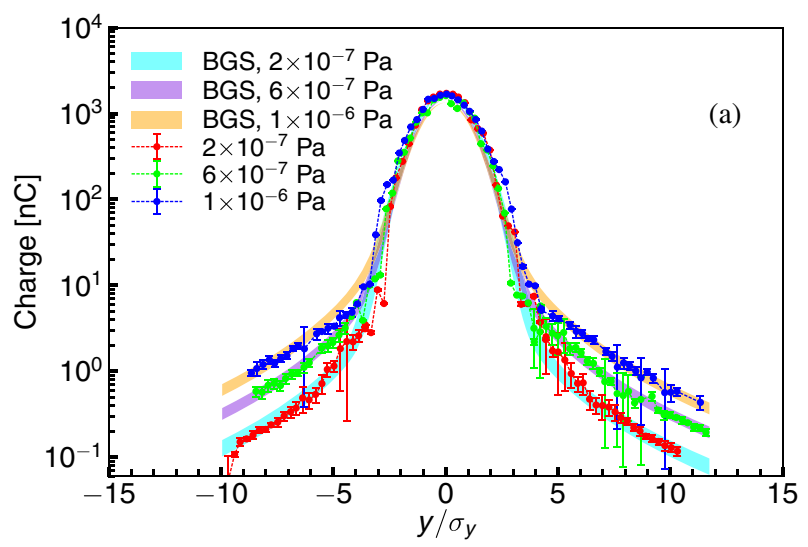

non-Gaussian tails in the energy distribution of the beam may also play a role.

\section{EMITTANCE GROWTH FROM BEAM GAS SCATTERING}

Large-angle scattering events are rare but can induce large betatron oscillation amplitudes, which drive particles into the halo region. Small-angle scattering events have higher probability and will act analogously to quantum excitation. They can dilute the core particle distribution and cause emittance growth.

For typical vacuum pressures $\left(10^{-7}-10^{-6} \mathrm{~Pa}\right)$ at ATF, vertical emittance dilution is estimated with the beam distribution function derived in Sec. I and using Monte Carlo simulation. We assume that the worst vacuum pressure is $5 \times 10^{-6} \mathrm{~Pa}$ and the equilibrium vertical emittance (without BGS and IBS) is $12.8 \mathrm{pm}$. This value is increased to $18.4 \mathrm{pm}$ and $18.9 \mathrm{pm}$, as predicted by the analytic approximation and Monte Carlo simulation (see Fig. 10), respectively.

To further probe the above predictions, measurements of vertical emittance were performed for vacuum pressures ranging from $2.5 \times 10^{-7} \mathrm{~Pa}$ to $1.75 \times 10^{-6} \mathrm{~Pa}$. Vertical emittance was evaluated from the beam size measured by an X-ray synchrotron radiation (XSR) monitor and the corresponding $\beta$ function [29]. The observed vertical emittance increases from $12.63 \pm 0.46 \mathrm{pm}$ to $16.02 \pm 0.98 \mathrm{pm}$, which is higher than the simulation result, see Fig. 11(a). The difference might be caused by the uncertainty in the vacuum pressure measurement, systematic errors in the XSR monitor or some other physical process contributing to emittance growth [30]. Moreover, the vertical beam size monitored by the XSR reduces from $7.02 \mu \mathrm{m}$ to $6.2 \mu \mathrm{m}$ when the vacuum pressure recovers from $1.75 \times 10^{-6} \mathrm{~Pa}$ to $2.5 \times 10^{-7} \mathrm{~Pa}$, as shown in Fig. 11(b). This evidence indicates that emittance growth due to BGS is also visible for typical vacuum pressures of

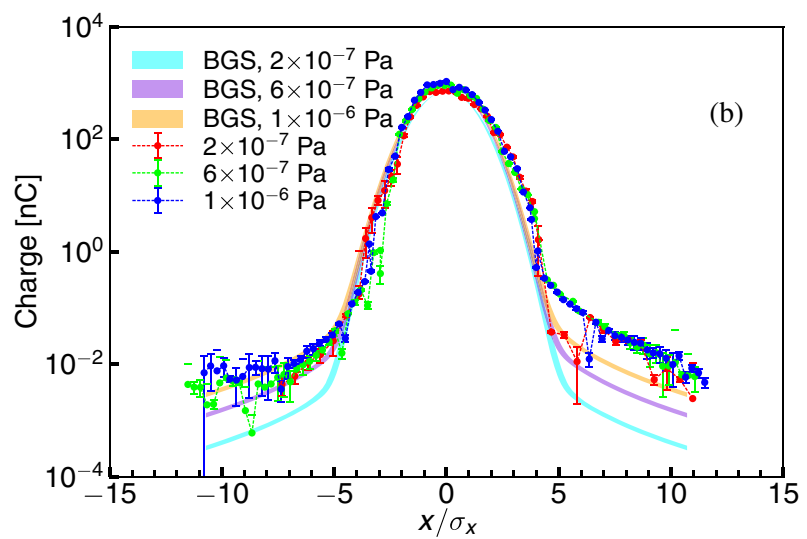

FIG. 9. Vertical (a) and horizontal (b) beam profiles normalized to beam core sizes for different vacuum pressures. The widths of the bands shown for the predictions from the BGS simulation represent the uncertainty of the beam size measurement. 


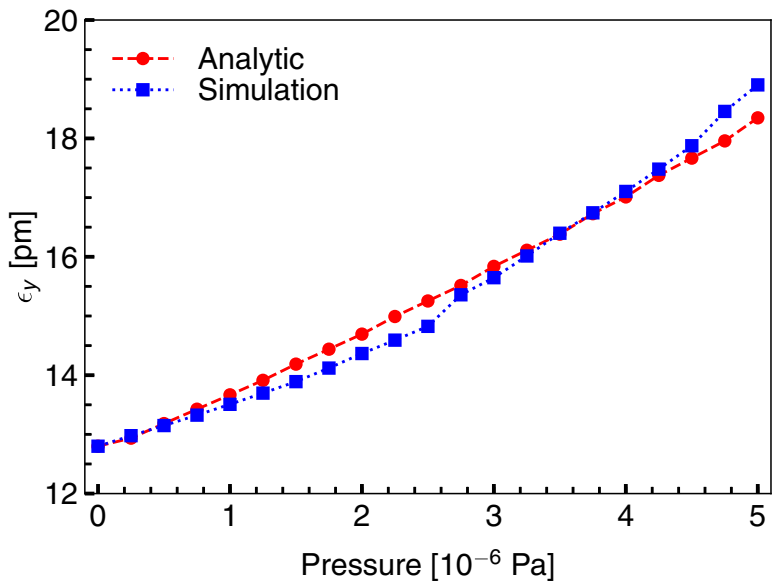

FIG. 10. Emittance growth as a function of vacuum pressure predicted by analytic calculation and Monte Carlo simulation.
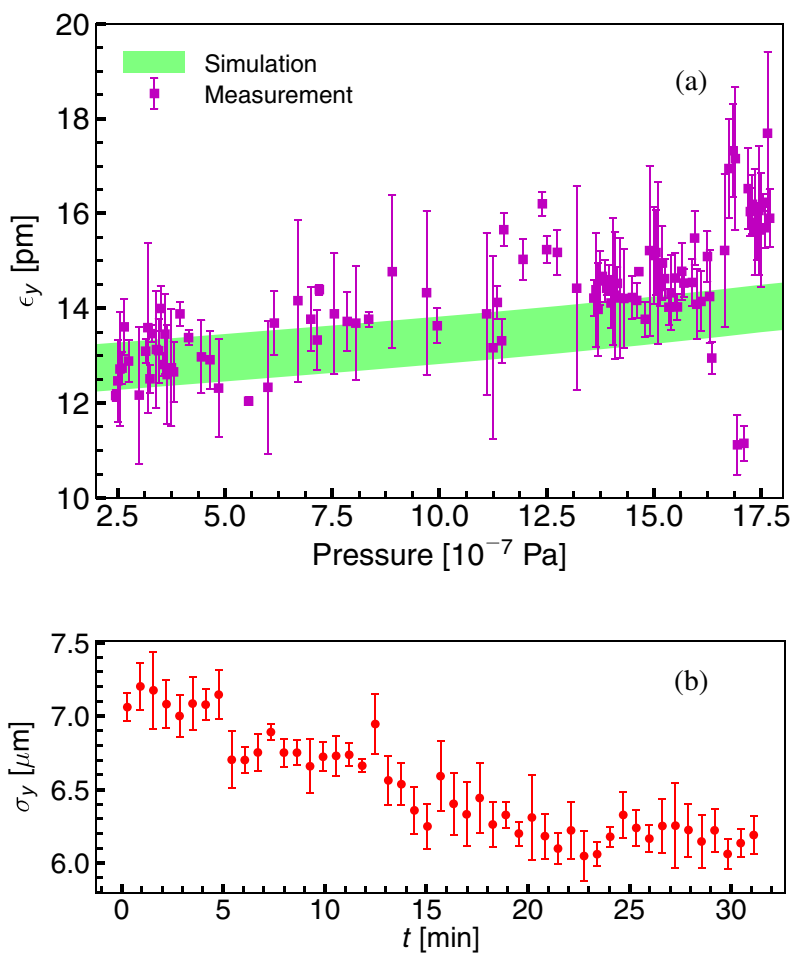

FIG. 11. Evaluation of vertical emittance with respect to vacuum pressure of the ring (a) and evolution of beam size measured by XSR when all SIPs were reset at $t=0$ (b). Band width of the vertical emittance estimated by simulation is due to the $10 \%$ uncertainty for the determination of the vertical emittance in absence of BGS.

$\sim 10^{-6} \mathrm{~Pa}$ and should be taken into account in the design of low-emittance storage ring.

\section{DISCUSSION AND CONCLUSIONS}

To explore the primary mechanisms of halo formation at ATF, systematic analytical calculations, simulations and experimental measurements have been carried out. We applied formulas to approximate the beam distribution function in the presence of radiation damping, quantum excitation and BGS in the normalized coordinate system. The simplified formalism, Eq. (15), is suitable for the estimation of beam halo, and also the beam core dilution.

For accurate predictions of the beam distribution distortion, a detailed Monte Carlo simulation was developed in the context of the SAD program. The actual COD and equilibrium beam parameters were modeled by introducing local orbit bumps using steering magnets. We attempted to benchmark the simulations using the vacuum lifetime, which was found to be 83 and $87 \mathrm{~min}$, for the two numerical methods, respectively, while the measured value was $16 \mathrm{~min}$. The presence of additional horizontal beam halo, from sources other than BGS, and the reduced dynamic aperture due to nonlinear fields, e.g., sextupole and octupole fields near pole-tip of quadrupole magnets, and high-order components at the extrance and exit of combined function bending magnets, may be reasons for this difference [31].

To extend the dynamic range of the diamond sensor detector used for the halo measurements, a rescaling scheme based on self-calibration was applied to the DS data. After the rescaling correction, an effective dynamic range of $10^{5}$ was achieved. Vertical and horizontal beam halo were measured for several vacuum pressures. For the vertical halo, good agreement between numerical estimations and experimental results for the different vacuum levels was observed. This clearly showed that the vertical halo is dominated by elastic BGS in the ring. On the other hand, the horizontal halo measured by the DS is higher than the BGS prediction and found to be asymmetric. The change in horizontal halo as a function of vacuum pressure is also negligible. This shows that BGS has almost no influence on the horizontal beam halo and other processes (e.g. chromaticity, Touschek scattering, and resonances) may play important roles.

Simulations and experimental observations of the vertical beam distribution clearly demonstrate that, for typical vacuum pressures in the ATF damping ring, halo generation, and emittance growth due to BGS are both measurable and significant.

Further studies of beam halo formation at the ATF have been proposed, including the installation of a new YAG/Optical Transition Radiation (OTR) monitor at a dispersion-free region after extraction from the ring, halo measurements for different kicker timings and optical focusing, and investigation of tails in the momentum distribution.

\section{ACKNOWLEDGMENTS}

The authors express their gratitude to the ATF Collaboration and to the staff and engineers of ATF. One of us (R. Yang) would like to particularly thank K. Oide for 
the support and advice on the proper usage of the SAD software, offering suggestions and encouragement. This work was supported by the Chinese Scholarship Council, the Toshiko Yuasa France-Japan Particle Physics Laboratory (Project No. A-RD-10), the France-China Particle Physics Laboratory (Project No. DEV-IHEP-LAL-LC-CEPC) and the MSCA-RISE E-JADE project, funded by the European Commission under Grant No. 645479.

[1] G. Stancari, A. Valishev, G. Annala, G. Kuznetsov, V. Shiltsev, D. A. Still, and L. G. Vorobiev, Collimation with Hollow Electron Beams, Phys. Rev. Lett. 107, 084802 (2011).

[2] C. Allen et al., Beam-Halo Measurements in High-Current Proton Beams, Phys. Rev. Lett. 89, 214802 (2002).

[3] P. Tenenbaum, T. Raubenheimer, and M. Woodley, in Proceedings of the 19th Particle Accelerator Conference, Chicago, IL, 2001 (IEEE, Piscataway, NJ, 2001), p. 3843.

[4] J. Qiang and R. D. Ryne, Beam halo studies using a threedimensional particle-core model, Phys. Rev. ST Accel. Beams 3, 064201 (2000).

[5] T. P. Wangler et al., Beam-halo in mismatched proton beams, Nucl. Instrum. Methods Phys. Res., Sect. A 519, 425 (2004).

[6] K. Wittenburg, CERN Accelerator School 2009-005, 2009, p. 557.

[7] J. Yan, Y. Yamaguchi, Y. Kamiya, S. Komamiya, M. Oroku, T. Okugi, N. Terunuma, K. Kubo, T. Tauchi, and J. Urakawa, Measurement of nanometer electron beam sizes with laser interference using Shintake Monitor, Nucl. Instrum. Methods Phys. Res., Sect. A 740, 131 (2014).

[8] K. Hirata and K. Yokoya, NonGaussian distribution of electron beams due to incoherent stochastic processes, Part. Accel. 39, 147 (1992).

[9] S. Liu, Ph.D. thesis, Universite Paris-Sud, 2015.

[10] T. Naito and T. Mitsuhashi, in Proceedings of IBIC2015, Melbourne, Australia (2016), p. 373.

[11] D. Wang, J. Gao, P. Bambade, and K. Yokoya, Analytical estimation of ATF beam halo distributio, Chin. Phys. C 38, 127003 (2014).

[12] S. Liu et al., In vacuum diamond sensor scanner for beam halo measurements in the beam line at the KEK Accelerator Test Facility, Nucl. Instrum. Methods Phys. Res., Sect. A 832, 231 (2016).
[13] Y. Honda et al., Achievement of Ultralow Emittance Beam in the Accelerator Test Facility Damping Ring, Phys. Rev. Lett. 92, 054802 (2004).

[14] T. Okugi et al., Linear and second order optics corrections for the KEK Accelerator Test Facility final focus beam line, Phys. Rev. ST Accel. Beams 17, 023501 (2014).

[15] F. Hinode et al., KEK Internal Report No. 95, 1995.

[16] K. Kubo et al., Extremely Low Vertical-Emittance Beam in the Accelerator Test Facility at KEK, Phys. Rev. Lett. 88, 194801 (2002).

[17] P. Raimondi and A. Seryi, Novel Final Focus Design for Future Linear Colliders, Phys. Rev. Lett. 86, 3779 (2001).

[18] G. White et al., Experimental Validation of a Novel Compact Focusing Scheme for Future Energy-Frontier Linear Lepton Colliders, Phys. Rev. Lett. 112, 034802 (2014).

[19] K. Ohmi, K. Hirata, and K. Oide, From the beam-envelope matrix to synchrotron-radiation integrals, Phys. Rev. E 49, 751 (1994).

[20] T. Raubenheimer, Report No. KEK-92-7, 1992.

[21] S. O. Rice, Mathematical analysis of random noise, Bell Labs Technical Journal 23, 282 (1944).

[22] $\mathrm{SAD}$, is a computer program for accelerator design; see http://acc-physics.kek.jp/SAD/.

[23] K. Kubo, Simulation study of low emittance tuning of the Accelerator Test Facility damping ring at KEK, Phys. Rev. ST Accel. Beams 6, 092801 (2003).

[24] R. Yang, P. Bambade, K. Kubo, T. Okugi, N. Terunuma, and D. Zhou, Numerical investigation of beam halo from beam gas scattering in KEK-ATF, J. Phys. Conf. Ser. 874, 012063 (2017).

[25] W. Helmut, Particle Accelerator Physics (Third Edition) (Springer, Berlin, 2007), pp. 321-329.

[26] F. Zimmermann et al., Report No. SLAC-AP-113, 1998.

[27] T. Okugi, H. Hayano, K. Kubo, T. Naito, N. Terunuma, J. Urakawa, T. Hirose, F. Zimmermann, and T. O. Raubenheimer, Evaluation of vertical emittance in KEKATF by utilizing lifetime measurement, Nucl. Instrum. Methods Phys. Res., Sect. A 455, 207 (2000).

[28] V. Kubytskyi, S. Liu, and P. Bambade, in Proceedings of IPAC2015, Richmond, USA (2015), p. 787.

[29] T. Naito et al., in Proceedings of IBIC2012, Tsukuba, Japan (2012), p. 215.

[30] F. Zimmermann, Report No. SLAC-AP-107, 1997.

[31] F. Zimmermann, Report No. SLAC-PUB-7987, 1998. 